\title{
Human Cancers: The Interplay Between Protein-Coding Genes and Non-Coding RNAs
}

\author{
George A. Calin
}

Corresponding author: George A Calin, MD, PhD

E-mail: gcalin@mdanderson.org
Departments of Experimental Therapeutics and Leukemia, The University of Texas M. D. Anderson Cancer Center, Houston, TX, USA Center for RNA Interference and Non-Coding RNAs, The University of Texas MD Anderson Cancer Center, Houston, TX, USA

\section{ABSTRACT}

The discovery of non-coding RNAs (ncRNAs) dramatically changed the understanding of cancer mechanisms in the last decade. The ncRNAs interplay with protein-coding genes and their abnormalities represents one the most unexpected and important discoveries in the cancer field. Cancer initiation, progression and dissemination causally involve the effects of small regulatory ncRNAs named microRNAs, mainly due to deregulation of expression of cancer protein coding genes. miRNAs can act as oncogenes (activating malignant potential) or tumor suppressors (inhibiting malignant potential) directly on the tumor cells or via communication with tumor microenvironment cells. Understanding the roles of miRNAs and other ncRNAs in malignant cells uncovers a new layer of protein coding and non-coding gene regulation; furthermore, provides new markers for early diagnosis and improved prognosis, as well as novel therapeutics for cancer patients. Herein I will expose what is known about the miRNA function and describe examples and the challenges for clinical use of miRNAs in the near future.

Key words: microRNAs, oncogene, tumor suppressor, cancer, diagnosis, therapy

\section{microRNAs are short RNAs involved in physiologic and pathological processes}

MicroRNAs are defined as short non-coding RNAs (ncRNAs) of about 19 to 23 nucleotides (nt) in length, that are not translated in peptides but regulate protein coding gene expression at the posttranscriptional level (1). Precursor microRNAs in the form of hairpin loop structures, located in the nucleus, produce mature microRNAs that act at the cytoplasm level. The binding takes place at the target messenger RNAs (mRNAs) 3'untranslated regions (UTR), coding sequences or 5'UTR (mRNAs) (2). This leads to degradation of mRNA or translation inhibition with consequent target protein expression reduction. It is estimated that miRNAs regulate most part of the human genome, both protein coding as non-coding; recently, it was found that miRNAs could bind and block 
the function of longer non-coding RNAs (3). The importance of microRNAs in all physiological or pathological cellular processes is supported by the fact that their structure is highly conserved among orthologous species. Cell cycle regulation, immune system functionality, apoptosis or cell death, cellular aging, differentiation, metabolism and neuronal patterning are all regulated by these short ncRNAs $(4,5)$.

\section{microRNAs alterations are identified in all human cancers analyzed to date}

Normal levels of expression of mature and/or precursor miRNAs in normal cells versus the abnormal malignant cells represent the main mechanism of microRNoma (the full spectrum of microRNAs from human genome) alterations (table 1) (6). This is due to numerous reversible or irreversible altered mechanisms, such as the epigenetic regulation of miRNA expression, microRNAs mutated loci, the location of miRNAs at aberrant cancer genomic regions, or defects in miRNA processing proteins including mutations in Dicer (an endoribonuclease involved in the production of mature miRNAs) or Exportin 5 (a protein involved in transport of pre-microRNA out of the nucleus) $(7,8)$.

In 2002, Calin and colleagues reported for the first time miRNAs abnormalities in cancer: miR-15a and miR-16-1, that are located at the frequently deleted

Table 1 - Examples of oncogenic or suppressor microRNAs

Human MicroRNAs
let-7 family [TS]
(first family of miRs to prove
to be conserved between species)
miR-15a,miR-16-1 cluster [TS]
(first family of miRNAs to prove to
be abnormally expressed in cancer)

miR-17, miR-18a, miR-19a, miR-20a, miR-19b-1, miR-92a1, miR-17-92 cluster [OG] (first family of miRNAs proved to be oncogenic)

Examples of clinical correlations
Let-7b is reduced in leukemia (ALL, CLL), ovary
prostate, liver, brain cancers and correlates with
poor prognosis;
Lets-7i expression is reduced in breast, brain can
and associated with bad prognosis; let-7i affects
chemotherapy potency
Downregulated in CLL and associated with good
prognosis
High levels of miR-92a identified in leukemia,
(CLL, ALL) colorectal and ovary cancers.
Associates with poor prognosis.

(CLL, ALL) colorectal and ovary
Associates with poor prognosis. Examples of molecular mechanisms
Represses cell proliferation and growth
let-7f promotes angiogenesis
Targets: CCND1, CDC25a, CDC34,
CDK6, DICER, HMGA2, HOXA9, ITGB3,
MYC, RAS, TLR4

Induces apoptosis in leukemia cells miR-16 regulates cell cycle by downregulating G0/G1 proteins Targets: BCL2, CCND1, CDK6, CDC27, HMGA2, MCL1, MYB, VEGF, WNT3A miR-17, miR-18a, miR-19a, miR-20a, miR-19b-1

increase tumor growth and tumor vascularization; miR-20a is anti-apoptotic; Targets: AIB1 AML1, BIM1, E2F1, E2F2, E2F3, HIF-1A, PTEN, TGFBR2, TSP1

\section{miR-21 [OG]}

(the only microRNA identified overexpressed in any type of cancer analyzed)
Overexpressed in leukemia (CLL), liver, breast, colon, lung, pancreas, prostate, stomach, colorectal, brain, ovary, tongue, thyroid, uterine, head and neck cancers. Poor prognosis, associates with fludarabine refractory CLL
miR-21 knockdown induces apoptosis in glioblastoma miR-21 induces invasion, metastasis in colorectal cancer

Targets: BCL2, CDC25A, MASPIN, PDCD4, PTEN, TPM1, RECK, RASA1

\section{miR-34 family [TS]}

(first family to be used for cancer therapy in clinical trials)

Downregulated in leukemias and solid cancers and expression controlled by TP53 Low expression correlates with poor prognosis in leukemias

miR-34a induces downregulation of E2F in colon cancer Targets: BCL2, CCND1, CCNE2, CDK4, CDK6, C-MYC, DLL1, E23, HMGA2, MET, MYC, N-MYC, Notch1, SIRT1

(first miRNA to be proved oncogenic

\section{miR-155 [OG]} in an mouse transgenic model) Overexpressed in leukemia (CLL, AML), liver, breast, pancreas, lung, head and neck, thyroid, tongue carcinomas

Pre-B cell proliferation, lymphoblastic leukemia/high-grade lymphoma in miR-155 transgenic mice Targets: AGTR1, AID, FOX03A, IKBKE, SHIP-1, SOCS1, TP53INP1 Modulates mismatch-repair genes

\begin{tabular}{lll} 
miR-181 family [TS or OG] & Overexpression of miR-181a is reported in pancreas, & MYCN regulates transcription of miR-181 cluster \\
(first miRNA family to be involved & thyroid cancers, while downregulation in brain cancers. & Targets: HOXA11, TCL1 \\
in resistance to chemotherapy ) & $\begin{array}{l}\text { High miR-181a correlates with short interval from } \\
\text { diagnosis to therapy in CLL }\end{array}$ & \\
\hline
\end{tabular}

Note - TS - tumor suppressor role; OG - oncogene role; the gene symbols are as in NCBI at http://www.ncbi.nlm.nih.gov/. AML, acute myeloid leukemia; CLL, chronic lymphocytic leukemia; 
chromosome 13q14 in chronic lymphocytic leukemia (CLL) are deleted or downregulated in the almost all patients, indicating that these miRNAs can have a potential tumor suppressors roles (9). In a following report, the same group discovered that a significant number of miRNAs are located at genomic regions altered in cancers (such as amplifications or loss of heterozygosity or breakpoints), suggesting miRNAs as a new class of cancer genes (10). By developing the first microRNA microarray and by using large mutational screens, Calin and colleagues reported the first germline mutations in microRNAs as well as one of the earliest miRNA expression signature associated with cancer prognosis and evolution (11). Explaining the CLL phenotype, Cimmino and Calin and colleagues reported that both miR-15 and miR-16 are involved in programmed cell death through the targeting of the anti-apoptotic BCL2 messenger RNA (12). This induces low levels of BCL2 with consequent apoptosis and prolonged survival, the phenotypic characteristics of the malignant CD5/CD19-positive malignant CLL cells.

Further work from all around the world, reported in over 25,000 publications till date present days, deeply dissected the roles of miRNAs in tumors, both in malignant cells and in the tumor microenvironment cells (13). Also, mutations within microRNAs were identified as more prevalent in malignant cells as compared to normal cells, which shows that these mutations have a role in the malignant phenotype. An important number of studies revealed the dual role of microRNAs, that can exert a tumor suppressive role or, on the contrary, a tumor promoter (oncogenic) role: let-7 can be downregulated in lung and breast cancers and stimulate cell growth, while in same cancers, miR-17-92 if overexpressed leads to cancer growth (table 1) $(5,14)$. miR-15a and miR-16-1 reduced expression in prostate cancer promoted tumor growth and progression by the lack of repression of FGF-2 and its receptor FGFR1. Both FGF-2 and its receptor act on tumors cells as well as the surrounding stroma environment to enhance cancer cell survival, proliferation and metastasis and were identified as downregulated also in cancer-associated fibroblasts (CAFs) from the environment of prostate tumors (13).

\section{microRNA expression signatures from tumors or body fluids associates with patients' prognosis and overall survival}

The measurement of miRNAs in tumor tissues, plasma, serum and other body fluids represent a new exploratory road for noninvasive biomarkers in cancer
$(15,16)$. MicroRNA expression has been shown to forecast the clinical progression of cancers and other diseases. In CLL, both miR-29c and miR-223 are down regulated in patients who are predicted to have a poor prognosis with a shorter survival, while another important therapeutic candidate, miR-155 has been shown to be upregulated in the same category of patients. Also, patients with other hematological diseases like acute myeloid leukemia who present with high miR-191 expression have been reported to have a shorter survival time. Nevertheless, metastasis can be detected by using as biomarkers serum microRNAs known to influence many biological processes and secondary tumor development at additional locations in the body. The concerns about the high stability of miRNAs are mostly disproved by the findings that serum and plasma processing in severe conditions that would normally degrade most RNAs (such as boiling, extreme $\mathrm{pH}$ levels, or extended storage) keep the short RNAs unaltered (16). As an example, serum miR-21 levels were lower in hormone-refractory prostate cancer patients who responded to docetaxel-based chemotherapy versus those with resistant disease. Downregulation of let-7a, miR-17, and miR-34 family was correlated with sensitivity to 5-fluorouacil, adriamycin, or cyclophosphamide, all commonly used in various chemotherapy regimens (13). The use of miRNA biomarkers is not restricted only to cancer: for example, the same miR-155 and miR-223 have been implicated in Rheumatoid Arthritis (17). It was also demonstrated the miRNA aberrant patterns in cardiac hypertrophy and their roles analyzed, including that of miR-21, which is also one of the most deregulated miRNAs in cancer, suggesting common miRNA pathways involved in signaling pathways shared by both abnormal states (18).

\section{microRNA therapeutics available for cancer patients}

Contrary to chemotherapy, antisense oligonucleotides, small interfering RNAs, or small molecules, there is one major advantage of using miRNAs: they can target multiple genes from the same pathway significantly reducing the potential development of resistance due to multiple mutations in various genes from that specific pathway. For instance, miR-15a and miR-16-1, both with reduced expression in CLL, have two anti-apoptotic targets, the oncogenic messenger RNAs for MCL1 and BCL2. Targeting oncogenic miRNAs with anti-miRNAs or antagomiRs, or restoring the tumor suppressor miRNA levels by using miRNA mimics, although not the "universal panacea" for any type of cancer, could represent in 
the near future valid therapeutic options for specific categories of patients $(19,20)$.

In the first miRNA-targeting therapy to reach clinical trials in humans, antagomiR-122 (named Miravirsen) was well tolerated by individuals with of hepatitis $C$ (HCV) infection, a risk factor for developing hepatocellular carcinoma. The use of this antago-miR revealed mild side effects such as diarrhea or headache, being generally well tolerated by patients. Importantly, individuals treated with Miravirsen displayed a significant dose-dependent reduction in HCV RNA levels without any signs of viral resistance (21). A liposomeformulated mimic of the tumor suppressor miR-34a, called MRX34, in a Phase I clinical trial in patients with advanced solid tumors showed manageable toxicity profiles and strong evidence of activity in hepatocellular carcinomas, renal cell carcinomas and melanomas. Molecular analysis showed dose-dependent repression of miR-34a target oncogenes, including BCL2, CTNNB1 HDAC1, and FOXP1 in the tumors from the treated patients (19).

\section{PERSPECTIVES}

MiRNAs were discovered in 1993 and rapidly became an exciting topic of research during the last decade, with the number of published studies growing exponentially. miRNAs and other longer ncRNAs are involved not only in cancer-altered pathways but also in many other deadly diseases such as sepsis (22). Variations of the miRNome have been documented in cancer cells with respect to the normal cell counterpart. Similarly to microRNAs, other non-coding RNAs (such as circular RNAs or long intergenic non-codingRNAs) $(23,24)$ appear deregulated intumors. miRNAs and other ncRNAs have only recently been identified as new diagnostic and prognostic biomarkers for cancer evolution, and miRNAs based cancer therapy represents a treatment option already in medical practice that has to be tested for safety and efficacy.

\section{Acknowledgements}

Dr. Calin is The Alan M. Gewirtz Leukemia \& Lymphoma Society Scholar. Work in Dr. Calin's laboratory is supported in part by the $\mathrm{NIH} / \mathrm{NCl}$ grants 1UH2TR00943-01 and 1 R01 CA182905-01, the UT MD Anderson Cancer Center SPORE in Melanoma grant from $\mathrm{NCl}$ (P50 CA093459), Aim at Melanoma Foundation and the Miriam and Jim Mulva research funds, the UT MD Anderson Cancer Center Brain SPORE (2P50CA127001), a Developmental Research award from Leukemia SPORE, a CLL Moonshot Flagship project, a 2015 Knowledge GAP
MDACC grant, an Owens Foundation grant, and the Estate of C. G. Johnson, Jr. We included in the reference list mostly reviews, so the readers can use additional more detailed presentations of specific topics discussed in the present review.

\section{REFERENCES}

1. Ambros V. MicroRNA pathways in flies and worms: growth, death, fat, stress, and timing. Cell. 2003 Jun 13;113(6):673-6.

2. Bartel DP. MicroRNAs: genomics, biogenesis, mechanism, and function. Cell. 2004 Jan 23;116(2):281-97.

3. Gregory RI, Shiekhattar R. MicroRNA biogenesis and cancer. Cancer Res. 2005 May 1;65(9):3509-12.

4. Berezikov E, Plasterk RH. Camels and zebrafish, viruses and cancer: a microRNA update. Hum Mol Genet. 2005 Oct 15;14 Spec No. 2:R18390.

5. Tuna M, Machado AS, Calin GA. Genetic and epigenetic alterations of microRNAs and implications for human cancers and other diseases. Genes Chromosomes Cancer. 2016 Mar;55(3):193-214. doi: 10.1002/ gcc.22332. Epub 2015 Dec 9.

6. Berindan-Neagoe 11, Monroig Pdel C, Pasculli B, Calin GA. MicroRNAome genome: a treasure for cancer diagnosis and therapy. CA Cancer J Clin. 2014 Sep-0ct;64(5):311-36. doi: 10.3322/caac. 21244. Epub 2014 Aug 7.

7. Calin GA, Croce CM. MicroRNA signatures in human cancers. Nat Rev Cancer. 2006 Nov;6(11):857-66.

8. Esquela-Kerscher A, Slack FJ. OncomiRs - microRNAs with a role in cancer. Nat Rev Cancer. 2006 Apr;6(4):259-69.

9. Calin GA, Dumitru CD, Shimizu M, Bichi R, Zupo S, Noch E, et al. Frequent deletions and down-regulation of micro- RNA genes miR15 and miR16 at 13q14 in chronic lymphocytic leukemia. Proc Natl Acad Sci U S A. 2002 Nov 26;99(24):15524-9. Epub 2002 Nov 14.

10. Calin GA, Sevignani C, Dumitru CD, Hyslop T, Noch E, Yendamuri S, et al. Human microRNA genes are frequently located at fragile sites and genomic regions involved in cancers. Proc Natl Acad Sci U S A. 2004 Mar 2;101(9):2999-3004. Epub 2004 Feb 18.

11. Calin GA, Ferracin M, Cimmino A, Di Leva G, Shimizu M, Wojcik SE, et al. A MicroRNA signature associated with prognosis and progression in chronic lymphocytic leukemia. N Engl J Med. 2005 Oct 27;353(17): 1793-801.

12. Cimmino A, Calin GA, Fabbri M, lorio MV, Ferracin M, Shimizu M, et al. miR-15 and miR-16 induce apoptosis by targeting BCL2. Proc Natl Acad Sci U S A. 2005 Sep 27;102(39):13944-9. Epub 2005 Sep 15.

13. Berindan-Neagoe I, Calin GA. Molecular pathways: microRNAs, cancer cells, and microenvironment. Clin Cancer Res. 2014 Dec 15;20(24): 6247-53. doi: 10.1158/1078-0432.CCR-13-2500.

14. Nicoloso MS, Spizzo R, Shimizu M, Rossi S, Calin GA. MicroRNAs-the micro-steering wheel of tumor metastases. Nat Rev Cancer. 2009 Apr;9(4):293-302. doi: 10.1038/nrc2619. Epub 2009 Mar 5.

15. Cummins JM, Velculescu VE. Implications of micro-RNA profiling for cancer diagnosis. Oncogene. 2006 Oct 9;25(46):6220-7.

16. Cortez MA, Bueso-Ramos C, Ferdin J, Lopez-Berestein G, Sood AK, Calin GA. MicroRNAs in body fluids-the mix of hormones and biomarkers. Nat Rev Clin Oncol. 2011 Jun 7;8(8):467-77. doi: 10.1038 /nrclinonc.2011.76.

17. Churov AV, Oleinik EK, Knip M. MicroRNAs in rheumatoid arthritis: altered expression and diagnostic potential. Autoimmun Rev. 2015 Nov;14(11):1029-37. doi: 10.1016/j.autrev.2015.07.005. Epub 2015 Jul 8.

18. Small EM, Olson EN. Pervasive roles of microRNAs in cardiovascular biolobgy. Nature. 2011 Jan 20;469(7330):336-42. doi: 10.1038/ nature09783.

19. Ling $\mathrm{H}$, Fabbri M, Calin GA. MicroRNAs and other non-coding RNAs as targets for anticancer drug development. Nat Rev Drug Discov. 
2013 Nov;12(11):847-65. doi: 10.1038/nrd4140.

20. Monroig Pdel C, Chen L, Zhang S, Calin GA. Small molecule compounds targeting miRNAs for cancer therapy. Adv Drug Deliv Rev. 2015 Jan;81:104-16. doi: 10.1016/j.addr.2014.09.002. Epub 2014 Sep 17.

21. Janssen HL, Reesink HW, Lawitz EJ, Zeuzem S, Rodriguez-Torres M, Patel K, et al. Treatment of HCV infection by targeting microRNA. N Engl J Med. 2013 May 2;368(18):1685-94. doi: 10.1056/ NEJMoa1209026. Epub 2013 Mar 27.

22. Vasilescu C, Rossi S, Shimizu M, Tudor S, Veronese A, Ferracin M, et al. MicroRNA fingerprints identify miR-150 as a plasma prognostic marker in patients with sepsis. PLoS One. 2009 0ct 12;4(10):e7405. doi: 10.1371/journal.pone.0007405.

23. Taft RJ1, Pang KC, Mercer TR, Dinger M, Mattick JS. Non-coding RNAs: regulators of disease. J Pathol. 2010 Jan;220(2): 126-39. doi: 10.1002/path.2638.

24. Ling H, Vincent K, Pichler M, Fodde R, Berindan-Neagoe I, Slack FJ, et al. Junk DNA and the long non-coding RNA twist in cancer genetics. Oncogene. 2015 Sep 24;34(39):5003-11. doi: 10.1038/onc.2014.456. Epub 2015 Jan 26. 